\title{
Prevalence and predictors of depression among hemodialysis patients: a prospective follow-up study
}

\author{
Amjad Khan ${ }^{1,2,3^{*}}$, Amer Hayat Khan ${ }^{1,2^{*}}$, Azreen Syazril Adnan ${ }^{2,5}$, Syed Azhar Syed Sulaiman ${ }^{1}$ and Saima Mushtaq ${ }^{4}$
}

\begin{abstract}
Background: Even though depression is one of the most common psychiatric disorders, it is under-recognized in hemodialysis (HD) patients. Existing literature does not provide enough information on evaluation of predictors of depression among HD patients. The objective of the current study was to determine the prevalence and predictors of depression among HD patients.

Methods: A multicenter prospective follow-up study. All eligible confirmed hypertensive HD patients who were consecutively enrolled for treatment at the study sites were included in the current study. HADS questionnaire was used to assess the depression level among study participants. Patients with physical and/or cognitive limitations that prevent them from being able to answer questions were excluded.

Results: Two hundred twenty patients were judged eligible and completed questionnaire at the baseline visit. Subsequently, 216 and 213 patients completed questionnaire on second and final follow up respectively. The prevalence of depression among patients at baseline, 2nd visit and final visit was 71.3, 78.2 and 84.9\% respectively. The results of regression analysis showed that treatment given to patients at non-governmental organizations (NGO's) running $\mathrm{HD}$ centers $(\mathrm{OR}=0.347, p$-value $=0.039)$ had statistically significant association with prevalence of depression at final visit.

Conclusions: Depression was prevalent in the current study participants. Negative association observed between depression and hemodialysis therapy at NGO's running centers signifies patients' satisfaction and better depression management practices at these centers.
\end{abstract}

Keywords: Depression, HADS, Hemodialysis, Hypertension

\section{Background}

According to the guidelines of the World Health Organization (WHO), "depression is a common mental disorder, characterized by sadness, loss of interest or pleasure, feelings of guilt or low self-worth, disturbed sleep or appetite, feelings of tiredness and poor concentration" [1]. Among end stage renal disease (ESRD) patient's depression is one of the most common psychiatric disorders [2]. The prevalence of depression is known to be much higher in HD patients as compared to other individuals of normal population [3]. Like in other chronic

\footnotetext{
* Correspondence: amjadkhan@qau.edu.pk; dramer2006@gmail.com

${ }^{1}$ Discipline of Clinical Pharmacy, School of Pharmaceutical Sciences, Universiti

Sains Malaysia, 11800 Penang, Malaysia

Full list of author information is available at the end of the article
}

disease conditions and in general population, evidence does exist that depression in patients on hemodialysis is associated with mortality $[4,5]$. It is under-recognized in HD patients because healthcare providers giving facilities, treatment and routinely work with these patients cannot give attention to control depression due to the nature of their illness [2]. There is a need of regular implementation of screening of depression among this population. Depression and anxiety both are strongly associated with patient's quality of life (QOL). One study suggests that depression among divorced and widowed women strongly affected patient's QOL [6].

Different questionnaires have been compiled and tested to investigate and measure the problems of 'anxiety and depression' commonly found in ESRD patients, 
including to those of "Hospital Anxiety and Depression Scale (HADS)" and "Beck's Depression Inventory (BDI)", both being properly validated in chronic kidney disease (CKD) patients [7]. The former questionnaire (HADS) was developed with the objective to investigate anxiety and depression associated fresh cases in an adult population. The later (HADS) one is different than the former one as it was developed to address the symptomatic position with respect to anxiety and depression. It is known that HD patients have higher rates of depression prevalence in contrast to the PD patients. The possible reasons are because HD patients usually stay connected with the machine during dialysis procedure which directly affects their daily activities and independence. It has also been reported that among the HD patients suicide rates are much higher. Moreover, it is found that due to the give flexibility and because of limited restrictions in their diet and social activities PD patients live with better quality of life [8-10].

To identify the factors associated with depression and anxiety, there is need of to conduct appropriate longitudinal studies. The instant research work was carried out to determine the contributing action of such factors in causing depression among HD population. Moreover, the expected outcomes of this study will give a comparative information on better management practices of depression at different dialysis facilities.

\section{Methods}

\section{HADS questionnaire}

HADS has been used for numerous studies among HD patients and showed acceptable reliability and validity [7]. Zigmond and Snaith are the original developers of HADS [11]. This scale cannot be used as a clinical diagnostic tool [12]. HADS has many advantages in terms of its interpretability (the results are very easy to interpret), in terms of its acceptability (widely accepted and can be used in a number of populations), in terms of its feasibility (the patients can complete the questionnaire within few minutes, no need of specialized training as the patients themselves can easily completed the questionnaire).

In the current study, we used the official validated Malay version of HADS provided by the original authors of the published Malay version of HADS from the department of psychiatry, Hospital Universiti Sains Malaysia (HUSM) [13].

\section{Administration of the HADS}

The total time required to complete the questionnaire is 2-5 min. Some cautions should be taken into consideration, for instance, the patients should be literate to read it. It can be a reasonable practice for the administrators of the HADS to ask patients first to read it once loudly and then fill it accordingly. HADS is comprised of 14 questions and have two domains: Anxiety (7 items) and depression (7 items). For Anxiety (HADS-A) this gave a specificity and sensitivity of 0.78 and 0.9 respectively. For depression (HADS-D) it gave a specificity and sensitivity of 0.79 and 0.83 respectively [14].

\section{Study design and setting}

This was a prospective follow-up study among HD patients conducted at HUSM and its affiliated dialysis centers. All eligible ( $>18$ years of age, literate and able to understand Malay) confirmed hypertensive HD patients who were consecutively enrolled for treatment at the study sites from 1st April 2017 to 31st December 2017 were included in the study. Patients with physical and/or cognitive limitations that prevent them from being able to answer questions were excluded.

\section{Data collection}

During the study period, all eligible HD patients who agreed to participate in the study by giving a written consent were asked to self-complete HADS questionnaire at three-time points: i) at baseline visit (initial evaluation), ii) after 3 months' interval (second follow up) and iii) at 6 months' interval (third follow up). Enrolled subjects who were unable to participate at the second follow up were not asked to take the questionnaire on third follow up. Using a standardized data collection form, socio-demographic and clinical data were collected from the regularly updated Advanced Dialysis Nephrology Application Network (ADNAN) at study sites. Height, weight and blood pressure were measured during a physical examination. Patient's interview and data abstraction tool designed by principal investigator based on an input from advisory committee, extensive literature review, hypothetical possible association and nephrologist's suggestions. At each interview session, the data collector evaluated the questionnaire for completion and asked the subject to provide missing response unless it was intentionally left unchecked.

\section{Scoring}

Grading on HADS questionnaire score sheet was used for scoring of questionnaires. Each question has 4 options; i) yes definitely (3), ii) yes sometimes (2), iii) No, not much (1), iv) No, not at all (0). For items $7 \& 10$ the scoring is reversed. Scores ranging on HADS from 0 to 7 are considered as non-case, score ranging from 8 to 10 is considered as borderline case and a score of $>11$ points were considered as case according to grading system of HADS. For the sake of analysis, we excluded borderline cases and only considered cases and non-cases. 


\section{Statistical analysis}

Statistical Package for Social Sciences (SPSS 21) was used for data analysis. Means and standard deviations were calculated for continuous variables, whereas categorical variable are presented as frequencies and percentages. Chi-squared test was used to observe significance between categorical variables. Multivariate logistic regression analysis with the Wald statistical criteria was used to obtain a final model. A $p$-value of < 0.05 was considered statistically significant. Relevant variables with a $p$-value $<0.25$ in the univariate analysis were included in the multivariate analysis [15]. We confirmed the correlations among variables entered in the multivariate analysis. The results of multivariate analysis were presented as beta, standard error, $P$-value, adjusted odds ratio and $95 \%$ confidence interval. The fit of the model was assessed by Hosmer Lemeshow and overall classification percentage.

\section{Results}

During the study recruitment period, a total of $272 \mathrm{HD}$ patients were enrolled for the treatment at the study sites. Fifty-two patients did not meet the eligibility criteria and were excluded. 220 patients were judged eligible and completed questionnaire at the baseline visit. Subsequently, 216 and 213 patients completed questionnaire on second and final follow up respectively (Fig. 1).

\section{Socio-demographic characteristics of patients evaluated for depression level}

The mean patient age was $56.58 \pm 11.09$ years. The majority of the patients were male (55.5\%), 41-60 years old (59.1\%), of a normal BMI (67.3\%), on dialysis for more than 5 years $(36.4 \%)$ and suffering from hypertension (91.8\%) "Table 1".

\section{Predictors of prevalence of depression among hemodialysis patients at baseline visit}

Table 2 shows that patients gender $(\mathrm{OR}=0.690, p$-value $=$ $0.224)$, socioeconomic status (high) $(\mathrm{OR}=0.500, p$-value $=0.182$ ), patients receiving treatment at NGO running $\mathrm{HD}$ centers $(\mathrm{OR}=0.508, p$-value $=0.105)$, patients receiving treatment at governmental $\mathrm{HD}$ centers $(\mathrm{OR}=0.475$, $p$-value $=0.030)$ and multitherapy $(\mathrm{OR}=0.659, p$-value $=$ $0.164)$ are the variables with $p$-value $<0.25$ and will be included in the multivariate analysis.

In Multivariate logistic regression analysis, the only variable which had statistically significant association

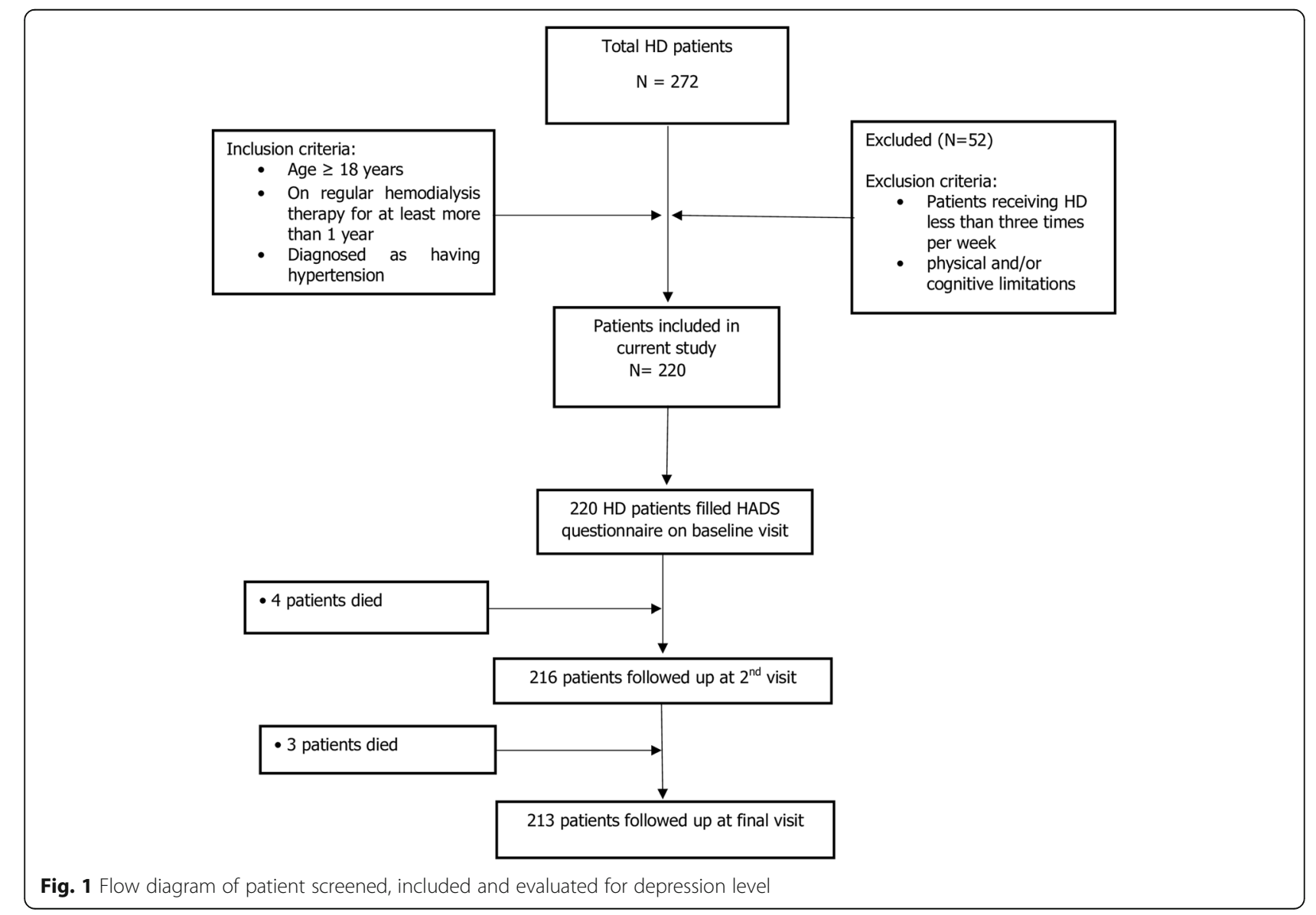


Table 1 Sociodemographics and clinical characteristics of patients $(N=220)$

\begin{tabular}{|c|c|}
\hline Variables & No. (\%) \\
\hline \multicolumn{2}{|l|}{ Gender } \\
\hline Female & $98(45.5)$ \\
\hline Male & $122(55.5)$ \\
\hline Age mean $( \pm S D)$ & $56.58( \pm 1$ \\
\hline \multicolumn{2}{|l|}{ Age group (years) } \\
\hline$<40$ & $17(7.7)$ \\
\hline $41-60$ & $130(59.1)$ \\
\hline$>60$ & $73(33.2)$ \\
\hline BMI mean $( \pm S D)$ & $23.57( \pm 4$ \\
\hline \multicolumn{2}{|l|}{ BMI Classification } \\
\hline Underweight & $12(5.5)$ \\
\hline Normal & $148(67.3)$ \\
\hline Overweight & $46(20.9)$ \\
\hline Obese & $14(6.4)$ \\
\hline \multicolumn{2}{|l|}{ Socioeconomic Status } \\
\hline Low & $39(17.7)$ \\
\hline Middle & $155(70.5)$ \\
\hline High & $26(11.8)$ \\
\hline \multicolumn{2}{|l|}{ Education Level } \\
\hline Uneducated & $74(33.6)$ \\
\hline Educated & $146(66.4)$ \\
\hline \multicolumn{2}{|l|}{ Marital Status } \\
\hline Single & $18(8.2)$ \\
\hline Married & $202(91.8)$ \\
\hline \multicolumn{2}{|l|}{ Race } \\
\hline Malay & $212(96.4)$ \\
\hline Others & $8(3.6)$ \\
\hline \multicolumn{2}{|l|}{ Smoking Status } \\
\hline Current Smoker & $73(33.2)$ \\
\hline Non-Smoker & $147(66.8)$ \\
\hline \multicolumn{2}{|l|}{ Alcohol } \\
\hline Current drinker & $18(8.2)$ \\
\hline Non-drinker & $202(91.8)$ \\
\hline \multicolumn{2}{|l|}{ Drug Addiction } \\
\hline Current Drug Addiction & $35(15.9)$ \\
\hline No Drug Addiction & $185(84.1)$ \\
\hline \multicolumn{2}{|l|}{ Employment } \\
\hline Unemployed & $120(54.5)$ \\
\hline Employed & $100(45.5)$ \\
\hline \multicolumn{2}{|l|}{ Dialysis Years } \\
\hline 1 year & $62(28.2)$ \\
\hline $2-4$ years & $78(35.5)$ \\
\hline$>5$ years & $80(36.4)$ \\
\hline
\end{tabular}

Table 1 Sociodemographics and clinical characteristics of patients ( $N=220)$ (Continued)

\begin{tabular}{ll}
\hline Variables & No. (\%) \\
\hline Hemodialysis Centers & $129(58.6)$ \\
Private & $33(15)$ \\
NGO & $58(26.4)$ \\
Governmental & \\
Vascular access & $204(92.7)$ \\
Fistula & $16(7.3)$ \\
Others & \\
Hypertension & $18(8.2)$ \\
No & $202(91.8)$ \\
Yes & \\
Diabetes Mellitus & $81(36.8)$ \\
No & $139(63.2)$ \\
Yes & \\
Cardiovascular Diseases & $185(84.1)$ \\
No & $35(15.9)$ \\
Yes
\end{tabular}

Other Comorbidities including: Blood clots, depression, asthma, osteoarthritis, pregnancy losses/birth defects and osteoporosis. Low socioeconomic status ( $\leq$ RM 2300 or 531 USD), Middle socioeconomic status (RM 2301-5600 or 5311294 USD) and High socioeconomic status (> RM 5600 or 1294 USD)

$S D$ Standard deviation, $B M I$ Body Mass Index

with prevalence of depression at baseline visit was treatment given to patients at NGO's running HD centers $(\mathrm{OR}=0.413, p$-value $=0.046)($ Table 2$)$.

\section{Predictors of prevalence of depression among hemodialysis patients at 2 nd visit}

Table 3 shows that patients' gender $(\mathrm{OR}=0.676$, $p$-value $=0.245$ ), treatment at NGO's running HD centers $(\mathrm{OR}=0.519, \quad p$-value $=0.139), \quad$ Diabetes $\quad(\mathrm{OR}=$ $0.646, p$-value $=0.219)$ and multi-therapy $(\mathrm{OR}=0.653$, $p$-value $=0.198)$ are the variables with $p$-value $<0.25$ and will be included in the multivariate analysis.

In multivariate logistic regression analysis, no significant association was found between depression and any sociodemographic and clinical factors (Table 3).

\section{Predictors of prevalence of depression among} hemodialysis patients at final visit

Analysis of prevalence of depression at final visit presented in (Table 4) revealed that BMI (normal) $(\mathrm{OR}=$ 4.133, $p$-value $=0.039)$, BMI (overweight $)(\mathrm{OR}=5.333$, $p$-value $=0.037)$, treatment given at NGO's running HD centers $(\mathrm{OR}=0.334, p$-value $=0.030)$, treatment given at governmental HD centers $(\mathrm{OR}=0.485, p$-value $=0.105)$, gouty arthritis $(\mathrm{OR}=2.630, p$-value $=0.203)$ are the variables with $p$-value $<0.25$ and will be included in the multivariate analysis. 
Table 2 Predictors of prevalence of depression among hemodialysis patients at baseline visit $(n=220)$

\begin{tabular}{|c|c|c|c|c|c|c|}
\hline \multirow[t]{2}{*}{ Variables } & \multicolumn{2}{|c|}{ Prevalence of Depression (No. \%) } & \multirow{2}{*}{$\begin{array}{l}\text { Univariate analysis } \\
\text { OR ( } 95 \% \mathrm{CI})\end{array}$} & \multirow[t]{2}{*}{$P$-value } & \multirow{2}{*}{$\begin{array}{l}\text { Multivariate analysis } \\
\text { OR ( } 95 \% \mathrm{Cl})\end{array}$} & \multirow[t]{2}{*}{$P$-value } \\
\hline & No & Yes & & & & \\
\hline \multicolumn{7}{|l|}{ Gender } \\
\hline Female & $23(23.4)$ & $75(76.6)$ & Referent & & Referent & \\
\hline Male & $40(32.8)$ & $82(67.2)$ & $0.690(0.380-1.254)$ & 0.224 & $0.742(0.399-1.383)$ & 0.348 \\
\hline \multicolumn{7}{|l|}{ Age (years) } \\
\hline$\leq 40$ & $4(23.5)$ & $13(76.5)$ & Referent & & & \\
\hline $41-60$ & $40(30.8)$ & $90(69.2)$ & $0.692(0.213-2.255)$ & 0.542 & & \\
\hline$>60$ & $19(26)$ & $54(74)$ & $0.874(0.254-3.012)$ & 0.832 & & \\
\hline \multicolumn{7}{|l|}{ BMI } \\
\hline Underweight & $4(33.3)$ & $8(66.7)$ & Referent & & & \\
\hline Normal & $41(27.7)$ & $107(72.3)$ & $1.305(0.373-4.568)$ & 0.677 & & \\
\hline Overweight & $11(23.9)$ & $35(76.1)$ & $1.591(0.401-6.313)$ & 0.509 & & \\
\hline Obese & $7(50)$ & $7(50)$ & $0.500(0.102-2.460)$ & 0.394 & & \\
\hline \multicolumn{7}{|l|}{ Socioeconomic Status } \\
\hline Low & $13(33.3)$ & $26(66.7)$ & Referent & & Referent & \\
\hline Middle & $37(23.9)$ & $118(76.1)$ & $1.595(0.745-3.414)$ & 0.230 & $1.826(0.816-4.086)$ & 0.143 \\
\hline High & $13(50)$ & $13(50)$ & $0.500(0.181-1.382)$ & 0.182 & $0.570(0.194-1.677)$ & 0.307 \\
\hline \multicolumn{7}{|l|}{ Marital Status } \\
\hline Single & $4(22.2)$ & $14(77.8)$ & Referent & & & \\
\hline Married & $59(29.2)$ & $143(70.8)$ & $0.692(0.219-2.191)$ & 0.532 & & \\
\hline \multicolumn{7}{|l|}{ Race } \\
\hline Malay & $62(29.2)$ & $150(70.8)$ & Referent & & & \\
\hline Others & $1(12.5)$ & $7(87.5)$ & $2.893(0.349-24.011)$ & 0.325 & & \\
\hline \multicolumn{7}{|l|}{ Smoking status } \\
\hline Current Smoker & $21(28.8)$ & $52(71.2)$ & Referent & & & \\
\hline Non-Smoker & $42(28.6)$ & $105(71.4)$ & $1.010(0.543-1.877)$ & 0.976 & & \\
\hline \multicolumn{7}{|l|}{ Alcohol } \\
\hline Current drinker & $6(33.3)$ & $12(66.7)$ & Referent & & & \\
\hline Non-drinker & $57(28.2)$ & $145(71.8)$ & $1.272(0.456-3.551)$ & 0.646 & & \\
\hline \multicolumn{7}{|l|}{ Drug Addiction } \\
\hline Current Drug Addiction & $8(22.9)$ & $27(77.1)$ & Referent & & & \\
\hline No Drug Addiction & $55(29.7)$ & $130(70.3)$ & 0.700 (0.299-1.638) & 0.411 & & \\
\hline \multicolumn{7}{|l|}{ Employment } \\
\hline Unemployed & $32(26.7)$ & $88(73.3)$ & Referent & & & \\
\hline Employed & $31(31)$ & $69(69)$ & $0.809(0.451-1.454)$ & 0.479 & & \\
\hline \multicolumn{7}{|l|}{ Dialysis Years } \\
\hline 1 year & $23(37.1)$ & $39(62.9)$ & Referent & & & \\
\hline $2-4$ years & $21(26.9)$ & $57(73.1)$ & $1.601(0.781-3.283)$ & 0.299 & & \\
\hline$>5$ years & $19(23.8)$ & $61(76.3)$ & $1.893(0.914-3.923)$ & 0.686 & & \\
\hline \multicolumn{7}{|l|}{ Hemodialysis Centers } \\
\hline Private & $29(22.5)$ & $100(77.5)$ & Referent & & Referent & \\
\hline NGO & $12(36.4)$ & $21(63.6)$ & $0.508(0.223-1.153)$ & 0.105 & $0.413(0.173-0.985)$ & 0.046 \\
\hline Governmental & $22(37.9)$ & $36(62.1)$ & $0.475(0.242-0.930)$ & 0.030 & $0.522(0.248-1.100)$ & 0.087 \\
\hline
\end{tabular}


Table 2 Predictors of prevalence of depression among hemodialysis patients at baseline visit $(n=220)$ (Continued)

\begin{tabular}{|c|c|c|c|c|c|c|}
\hline \multirow[t]{2}{*}{ Variables } & \multicolumn{2}{|c|}{ Prevalence of Depression (No. \%) } & \multirow{2}{*}{$\begin{array}{l}\text { Univariate analysis } \\
\text { OR }(95 \% \mathrm{Cl})\end{array}$} & \multirow[t]{2}{*}{$P$-value } & \multirow{2}{*}{$\begin{array}{l}\text { Multivariate analysis } \\
\text { OR }(95 \% \text { Cl) }\end{array}$} & \multirow[t]{2}{*}{$P$-value } \\
\hline & No & Yes & & & & \\
\hline \multicolumn{7}{|l|}{ Vascular access } \\
\hline Fistula & $59(28.9)$ & $145(71.1)$ & Referent & & & \\
\hline Others & $4(25)$ & $12(75)$ & $1.221(0.378-3.938)$ & 0.739 & & \\
\hline \multicolumn{7}{|l|}{ Diabetes Mellitus } \\
\hline No & $23(28.4)$ & $58(71.6)$ & Referent & 0.952 & & \\
\hline Yes & $40(28.8)$ & $99(71.2)$ & $0.981(0.535-1.800)$ & & & \\
\hline \multicolumn{7}{|c|}{ Cardiovascular Diseases } \\
\hline No & $55(29.7)$ & $130(70.3)$ & Referent & & & \\
\hline Yes & $8(22.9)$ & $27(77.1)$ & $1.428(0.611-3.339)$ & 0.411 & & \\
\hline \multicolumn{7}{|l|}{ Gouty Arthritis } \\
\hline No & $56(29.3)$ & $135(70.7)$ & Referent & & & \\
\hline Yes & $7(24.1)$ & $22(75.9)$ & $1.304(0.527-3.225)$ & 0.566 & & \\
\hline \multicolumn{7}{|c|}{ Other Comorbidities } \\
\hline No & $44(28.2)$ & $112(71.8)$ & Referent & & & \\
\hline Yes & $19(29.7)$ & $45(70.3)$ & $0.930(0.491-1.764)$ & 0.825 & & \\
\hline \multicolumn{7}{|l|}{ Type Therapy } \\
\hline Mono-therapy & $30(24.8)$ & $91(75.2)$ & Referent & & Referent & \\
\hline Multi-therapy & $33(33.3)$ & $66(66.7)$ & $0.659(0.366-1.186)$ & 0.164 & $0.553(0.293-1.043)$ & 0.067 \\
\hline
\end{tabular}

Analysis: Univariate and Multivariate binary logistic regression analysis. All variables with $p$-value $<0.25$ are included in the multivariate analysis Low socioeconomic status ( $\leq$ RM 2300 or 531 USD), Middle socioeconomic status (RM 2301-5600 or 531-1294 USD) and High socioeconomic status (> RM 5600 or 1294 USD)

OR Odds ratio, $\mathrm{Cl}$ confidence interval, $B M I$ Body mass index, NGO Non-governmental organization

Other comorbidities: Blood clots, depression, asthma, osteoarthritis, pregnancy losses/birth defects and osteoporosis

Table 4 shows that in multivariate logistic regression analysis, treatment given to patients at NGO's running $\mathrm{HD}$ centers $(\mathrm{OR}=0.347, p$-value $=0.039)$ had statistically significant association with prevalence of depression at final visit.

\section{Discussion}

To the best of our knowledge, this is the first follow up study which evaluated the prevalence and factors associated with depression among HD patients in Malaysia. In the current study, 220 eligible patients filled the HADS questionnaire on baseline and 213 filled it at the end of the study.

In our study $157(71.3 \%)$ patients suffered from depression at baseline, 169 (78.2\%) on 2nd evaluation and $181(84.9 \%)$ on the final visit respectively. However, the previously published literature has reported a comparatively low rate of depression among HD patients, ranging from 23.3 to $60.5 \%$ [2, 16-25].

In our study the rate of depression worsened with the passage of time, a linear increase was found from baseline $(71.3 \%)$ to final visit $(84.9 \%)$ among patients. The possible reasons for this finding could be the lifelong dialysis therapy with at least 3 dialysis procedures per week, patients taking too much medicine at once, economic burden on patients and their families and altered family and social relationships. Similar findings were reported in various studies where depression was noted to increase from baseline towards the end of the study period [18, 26, 27]. Keskin et al. revealed that depression is a risk factor for suicidal ideation and the chances of suicide attempts increasing with the severity of depression. Therefore, HD patients should be under regular psychiatric evaluation and all risk factors should be properly evaluated [28]. Depressive symptoms were linearly increasing in a population of chronic HD patients and there was a significant association of poor sleep quality, unemployment, pruritus, hypoalbuminemia and diabetes with depressive symptoms. Women were at increased risk of depression [29].

There is a wealth of evidence that dialysis has negative impact on depression and the severe depression among patients is in turn associated with mortality among these patients. Fifteen large scales studies indicating the significant association of depression with mortality among dialysis patients [30]. Significantly higher mortality risks were observed with depressive symptoms in patients on dialysis therapy in various longitudinal studies that assessed the repeated measurement of depression [3133]. Studies indicated that depression is associated with 
Table 3 Predictors of prevalence of depression among hemodialysis patients at 2 nd visit $(n=216)$

\begin{tabular}{|c|c|c|c|c|c|c|}
\hline \multirow[t]{2}{*}{ Variables } & \multicolumn{2}{|c|}{ Prevalence of Depression (No. \%) } & \multirow{2}{*}{$\begin{array}{l}\text { Univariate analysis } \\
\text { OR ( } 95 \% \mathrm{Cl})\end{array}$} & \multirow[t]{2}{*}{$P$-value } & \multirow{2}{*}{$\begin{array}{l}\text { Multivariate analysis } \\
\text { OR }(95 \% \mathrm{Cl})\end{array}$} & \multirow[t]{2}{*}{$P$-value } \\
\hline & No & Yes & & & & \\
\hline \multicolumn{7}{|l|}{ Gender } \\
\hline Female & $17(17.3)$ & $81(82.7)$ & Referent & & & \\
\hline Male & $30(25.4)$ & $88(74.6)$ & $0.676(0.351-1.336)$ & 0.245 & $0.699(0.357-1.370)$ & 0.297 \\
\hline \multicolumn{7}{|l|}{ Age (years) } \\
\hline$\leq 40$ & $3(17.6)$ & $14(82.4)$ & Referent & & & \\
\hline $41-60$ & $28(22)$ & $99(78)$ & $0.758(0.203-2.824)$ & 0.679 & & \\
\hline$>60$ & $16(22.2)$ & $56(77.8)$ & $0.750(0.192-2.937)$ & 0.680 & & \\
\hline \multicolumn{7}{|l|}{ BMI } \\
\hline Underweight & $4(33.3)$ & $8(66.7)$ & Referent & & & \\
\hline Normal & $28(19.2)$ & $118(80.8)$ & $2.107(0.592-7.496)$ & 0.250 & & \\
\hline Overweight & $10(22.2)$ & $35(77.8)$ & $1.750(0.436-7.032)$ & 0.430 & & \\
\hline Obese & $5(38.5)$ & $8(61.5)$ & $0.800(0.155-4.123)$ & 0.790 & & \\
\hline \multicolumn{7}{|l|}{ Socioeconomic Status } \\
\hline Low & $10(25.6)$ & $29(74.4)$ & Referent & & & \\
\hline Middle & $29(19.1)$ & $123(80.9)$ & $1.463(0.641-3.337)$ & 0.366 & & \\
\hline High & $8(32)$ & $17(68)$ & $0.733(0.243-2.214)$ & 0.582 & & \\
\hline \multicolumn{7}{|l|}{ Marital Status } \\
\hline Single & $3(16.7)$ & $15(83.3)$ & Referent & & & \\
\hline Married & $44(22.2)$ & $154(77.8)$ & $0.700(0.194-2.528)$ & 0.586 & & \\
\hline \multicolumn{7}{|l|}{ Race } \\
\hline Malay & $47(22.6)$ & $161(77.4)$ & Non-computable & & & \\
\hline Others & - & $8(100)$ & & - & & \\
\hline \multicolumn{7}{|l|}{ Smoking status } \\
\hline Current Smoker & $15(21.1)$ & $56(78.9)$ & Referent & & & \\
\hline Non-Smoker & $32(22.1)$ & $113(77.9)$ & $0.946(0.474-1.889)$ & 0.875 & & \\
\hline \multicolumn{7}{|l|}{ Alcohol } \\
\hline Current drinker & $4(23.5)$ & $13(76.5)$ & Referent & & & \\
\hline Non-drinker & $43(21.6)$ & $156(78.4)$ & $1.116(0.346-3.598)$ & 0.854 & & \\
\hline \multicolumn{7}{|l|}{ Drug Addiction } \\
\hline Current Drug Addiction & $7(20.6)$ & $27(79.4)$ & Referent & & & \\
\hline No Drug Addiction & $40(22)$ & $142(78)$ & $0.920(0.373-2.269)$ & 0.857 & & \\
\hline \multicolumn{7}{|l|}{ Employment } \\
\hline Unemployed & $23(19.7)$ & $94(80.3)$ & Referent & & & \\
\hline Employed & $24(24.2)$ & $75(75.8)$ & $0.765(0.400-1.461)$ & 0.417 & & \\
\hline \multicolumn{7}{|l|}{ Dialysis Years } \\
\hline 1 year & $15(24.6)$ & $46(75.4)$ & Referent & & & \\
\hline $2-4$ years & $18(23.7)$ & $58(76.3)$ & $1.051(0.478-2.308)$ & 0.902 & & \\
\hline$>5$ years & $14(17.7)$ & $65(82.3)$ & $1.514(0.667-3.439)$ & 0.322 & & \\
\hline \multicolumn{7}{|l|}{ Hemodialysis Centers } \\
\hline Private & $23(18.4)$ & $102(81.6)$ & Referent & & Referent & \\
\hline NGO & $10(30.3)$ & $23(69.7)$ & $0.519(0.217-1.237)$ & 0.139 & $0.580(0.238-1.412)$ & 0.580 \\
\hline Governmental & $14(24.1)$ & $44(75.9)$ & $0.709(0.334-1.504)$ & 0.370 & $0.646(0.295-1.417)$ & 0.276 \\
\hline
\end{tabular}


Table 3 Predictors of prevalence of depression among hemodialysis patients at 2nd visit $(n=216)$ (Continued)

\begin{tabular}{|c|c|c|c|c|c|c|}
\hline \multirow[t]{2}{*}{ Variables } & \multicolumn{2}{|c|}{ Prevalence of Depression (No. \%) } & \multirow{2}{*}{$\begin{array}{l}\text { Univariate analysis } \\
\text { OR }(95 \% \mathrm{Cl})\end{array}$} & \multirow[t]{2}{*}{$P$-value } & \multirow{2}{*}{$\begin{array}{l}\text { Multivariate analysis } \\
\text { OR ( } 95 \% \mathrm{Cl})\end{array}$} & \multirow[t]{2}{*}{$P$-value } \\
\hline & No & Yes & & & & \\
\hline \multicolumn{7}{|l|}{ Vascular access } \\
\hline Fistula & $44(22)$ & $156(78)$ & Referent & & & \\
\hline Others & $3(18.8)$ & $13(81.3)$ & $1.222(0.333-4.481)$ & 0.762 & & \\
\hline \multicolumn{7}{|l|}{ Diabetes Mellitus } \\
\hline No & $14(17.3)$ & $67(82.7)$ & Referent & & Referent & \\
\hline Yes & $33(24.4)$ & $102(75.6)$ & $0.646(0.322-1.297)$ & 0.219 & $0.688(0.335-1.413)$ & 0.309 \\
\hline \multicolumn{7}{|c|}{ Cardiovascular Diseases } \\
\hline No & $40(22)$ & $142(78)$ & Referent & & & \\
\hline Yes & $7(20.6)$ & $27(79.4)$ & $1.087(0.441-2.679)$ & 0.857 & & \\
\hline \multicolumn{7}{|l|}{ Gouty Arthritis } \\
\hline No & $43(22.9)$ & $145(77.1)$ & Referent & & & \\
\hline Yes & $4(14.3)$ & $24(85.7)$ & $1.779(0.585-5.409)$ & 0.310 & & \\
\hline \multicolumn{7}{|c|}{ Other Comorbidities } \\
\hline No & $35(22.9)$ & $118(77.1)$ & Referent & & & \\
\hline Yes & $12(19)$ & $51(81)$ & $1.261(0.605-2.625)$ & 0.536 & & \\
\hline \multicolumn{7}{|l|}{ Type Therapy } \\
\hline Mono-therapy & $22(18.5)$ & $97(81.5)$ & Referent & & Referent & \\
\hline Multi-therapy & $25(25.8)$ & $72(74.2)$ & $0.653(0.341-1.250)$ & 0.198 & $0.628(0.319-1.237)$ & 0.178 \\
\hline
\end{tabular}

Analysis: Univariate and Multivariate binary logistic regression analysis. All variables with $p$-value $<0.25$ are included in the multivariate analysis Low socioeconomic status ( $\leq$ RM 2300 or 531 USD), Middle socioeconomic status (RM 2301-5600 or 531-1294 USD) and High socioeconomic status (> RM 5600 or 1294 USD)

OR Odds ratio, $\mathrm{Cl}$ confidence interval, BMI Body mass index, NGO Non-governmental organization

Other comorbidities: Blood clots, depression, asthma, osteoarthritis, pregnancy losses/birth defects and osteoporosis

initiation of early dialysis treatment $[34,35]$. Other studies found relationship of depression with immune and inflammatory responses [36, 37]. Previous studies revealed that poor nutrition and nonadherence to treatment is significantly linked with depression among HD patients [38, 39]. The findings of one other systematic review showed 2-fold risk of dying in patients with depression [40]. Additionally, age is also a risk factor of increased mortality in depressive patients. Findings of another study indicated that in depressive patients with age of 65 years or above, there is $41 \%$ higher risk of mortality [41]. Depression is common and serious psychiatric disorder but underrecognized in patients undergoing dialysis therapy. It is reported elsewhere that only one-third of the HD patients with a diagnosis of depression were receiving treatment [42, 43]. Only few observational studies and clinical trials demonstrated the outcomes with pharmacologic and non-pharmacologic therapies in depressive patients [4448]. Two systematic reviews of antidepressants use in treatment of depression among chronic renal failure patients concluded that the evidence for effectiveness of these drugs is insufficient $[49,50]$.

In our study, comparable rates of depression were observed among female (86.3\%) and male participants (83.9\%). In contrast to our finding of no significant association between rate of depression among male and female patients, a study conducted in the University of Michigan, female gender was a significant risk factor for depression [51]. Similar positive association between female gender and high rate of depression among HD patients have been reported elsewhere [52, 53]. On the other hand, in line with our finding, no significant differences were observed in prevalence of depression and life event variables among males and females study participants in a study conducted in Turkey [54]. In our study $86.6 \%$ patients with middle socioeconomic status were having depression. In a study conducted elsewhere, an inverse relation was observed between depression and socioeconomic status [55]. Similarly, in another study, poor quality of life and depression was reported in study participants with middle and low socioeconomic status [56]. In another study where authors were interested to determine the association between socioeconomic status and depression among community residents and psychiatric patients, the authors concluded that study subjects holding jobs were more likely to have depression as compared to jobless participants [57].

Of the total 195 married patients, 165 (84.6\%) were having depression in the current study. In contradiction to our study findings authors reported that depression 
Table 4 Predictors of prevalence of depression among hemodialysis patients at final visit $(n=213)$

\begin{tabular}{|c|c|c|c|c|c|c|}
\hline \multirow[t]{2}{*}{ Variables } & \multicolumn{2}{|c|}{ Prevalence of Depression (No. \%) } & \multirow{2}{*}{$\begin{array}{l}\text { Univariate analysis } \\
\text { OR (95\% CI) }\end{array}$} & \multirow[t]{2}{*}{$P$-value } & \multirow{2}{*}{$\begin{array}{l}\text { Multivariate analysis } \\
\text { OR }(95 \% \mathrm{Cl})\end{array}$} & \multirow[t]{2}{*}{$P$-value } \\
\hline & No & Yes & & & & \\
\hline \multicolumn{7}{|l|}{ Gender } \\
\hline Female & $13(13.7)$ & $82(86.3)$ & Referent & & & \\
\hline Male & $19(16.1)$ & $99(83.9)$ & $0.826(0.385-1.773)$ & 0.624 & & \\
\hline \multicolumn{7}{|l|}{ Age (years) } \\
\hline$\leq 40$ & $2(11.8)$ & $15(88.2)$ & Referent & & & \\
\hline $41-60$ & $20(15.6)$ & $108(84.4)$ & $0.720(0.153-3.394)$ & 0.678 & & \\
\hline$>60$ & $10(14.7)$ & $58(85.3)$ & $0.773(0.153-3.911)$ & 0.756 & & \\
\hline \multicolumn{7}{|l|}{ BMI } \\
\hline Underweight & $4(40)$ & $6(60)$ & Referent & & Referent & \\
\hline Normal & $20(13.9)$ & $124(86.1)$ & $4.133(1.071-15.951)$ & 0.039 & $3.339(0.833-13.376)$ & 0.089 \\
\hline Overweight & $5(11.1)$ & $40(88.9)$ & $5.333(1.110-25.636)$ & 0.037 & $4.205(0.834-21.187)$ & 0.082 \\
\hline Obese & $<5$ & $11(78.6)$ & $2.444(0.405-14.748)$ & 0.330 & $1.907(0.300-12.123)$ & 0.494 \\
\hline \multicolumn{7}{|l|}{ Socioeconomic Status } \\
\hline Low & $6(15.8)$ & $32(84.2)$ & Referent & & & \\
\hline Middle & $20(13.4)$ & $129(86.6)$ & 1.209 (0.449-3.258) & 0.707 & & \\
\hline High & $6(23.1)$ & $20(76.9)$ & $0.625(0.177-2.208)$ & 0.465 & & \\
\hline \multicolumn{7}{|l|}{ Marital Status } \\
\hline Single & $2(11.1)$ & $16(88.9)$ & Referent & & & \\
\hline Married & $30(15.4)$ & $165(84.6)$ & $0.688(0.150-3.145)$ & 0.629 & & \\
\hline \multicolumn{7}{|l|}{ Race } \\
\hline Malay & $32(15.6)$ & $173(84.4)$ & Non-computable & & & \\
\hline Others & - & $8(100)$ & & - & & \\
\hline \multicolumn{7}{|l|}{ Smoking status } \\
\hline Current Smoker & $12(16.4)$ & $61(83.6)$ & Referent & & & \\
\hline Non-Smoker & $20(14.3)$ & $120(85.7)$ & $1.180(0.541-2.573)$ & 0.677 & & \\
\hline \multicolumn{7}{|l|}{ Alcohol } \\
\hline Current drinker & $2(11.1)$ & $16(88.9)$ & Referent & & & \\
\hline Non-drinker & $30(15.4)$ & $165(84.6)$ & $0.688(0.150-3.145)$ & 0.629 & & \\
\hline \multicolumn{7}{|l|}{ Drug Addiction } \\
\hline Current Drug Addiction & $5(14.3)$ & $30(85.7)$ & Referent & & & \\
\hline No Drug Addiction & $27(15.2)$ & $151(84.8)$ & $0.932(0.332-2.615)$ & 0.894 & & \\
\hline \multicolumn{7}{|l|}{ Employment } \\
\hline Unemployed & $17(14.4)$ & $101(85.6)$ & Referent & & & \\
\hline Employed & $15(15.8)$ & $80(84.2)$ & $0.898(0.422-1.908)$ & 0.779 & & \\
\hline \multicolumn{7}{|l|}{ Dialysis Years } \\
\hline 1 year & $10(16.9)$ & $49(83.1)$ & Referent & & & \\
\hline $2-4$ years & $13(17.6)$ & $61(82.4)$ & $0.958(0.387-2.370)$ & 0.925 & & \\
\hline$>5$ years & $9(11.3)$ & $71(88.8)$ & $1.610(0.610-4.253)$ & 0.337 & & \\
\hline \multicolumn{7}{|l|}{ Hemodialysis Centers } \\
\hline Private & $13(10.4)$ & $112(89.6)$ & Referent & & Referent & \\
\hline NGO & $8(25.8)$ & $23(74.2)$ & $0.334(0.124-0.897)$ & 0.030 & $0.347(0.127-0.949)$ & 0.039 \\
\hline Governmental & $11(19.3)$ & $46(80.7)$ & $0.485(0.203-1.162)$ & 0.105 & $0.487(0.196-1.205)$ & 0.120 \\
\hline
\end{tabular}


Table 4 Predictors of prevalence of depression among hemodialysis patients at final visit $(n=213)$ (Continued)

\begin{tabular}{|c|c|c|c|c|c|c|}
\hline \multirow[t]{2}{*}{ Variables } & \multicolumn{2}{|c|}{ Prevalence of Depression (No. \%) } & \multirow{2}{*}{$\begin{array}{l}\text { Univariate analysis } \\
\text { OR }(95 \% \mathrm{Cl})\end{array}$} & \multirow[t]{2}{*}{$P$-value } & \multirow{2}{*}{$\begin{array}{l}\text { Multivariate analysis } \\
\text { OR }(95 \% \mathrm{Cl})\end{array}$} & \multirow[t]{2}{*}{$P$-value } \\
\hline & No & Yes & & & & \\
\hline \multicolumn{7}{|l|}{ Vascular access } \\
\hline Fistula & $29(14.6)$ & $169(85.4)$ & Referent & & & \\
\hline Others & $3(20)$ & $12(80)$ & $0.686(0.182-2.583)$ & 0.578 & & \\
\hline \multicolumn{7}{|l|}{ Diabetes Mellitus } \\
\hline No & $9(11.7)$ & $68(88.3)$ & Referent & & & \\
\hline Yes & $23(16.9)$ & $113(83.1)$ & $0.650(0.284-1.487)$ & 0.308 & & \\
\hline \multicolumn{7}{|c|}{ Cardiovascular Diseases } \\
\hline No & $29(16.2)$ & $150(83.8)$ & Referent & & & \\
\hline Yes & $3(8.8)$ & $31(91.2)$ & $1.998(0.572-6.973)$ & 0.278 & & \\
\hline \multicolumn{7}{|l|}{ Gouty Arthritis } \\
\hline No & $30(16.3)$ & $154(83.7)$ & Referent & & Referent & \\
\hline Yes & $2(6.9)$ & $27(93.1)$ & $2.630(0.594-11.653)$ & 0.203 & $2.637(0.577-12.056)$ & 0.211 \\
\hline \multicolumn{7}{|c|}{ Other Comorbidities } \\
\hline No & $24(16)$ & $126(84)$ & Referent & & & \\
\hline Yes & $8(19)$ & $55(87.3)$ & $1.310(0.554-3.096)$ & 0.539 & & \\
\hline \multicolumn{7}{|l|}{ Type Therapy } \\
\hline Mono-therapy & $16(13.8)$ & $100(86.2)$ & Referent & & & \\
\hline Multi-therapy & $16(16.5)$ & $81(83.5)$ & $0.810(0.382-1.719)$ & 0.583 & & \\
\hline
\end{tabular}

Analysis: Univariate and Multivariate binary logistic regression analysis. All variables with $p$-value $<0.25$ are included in the multivariate analysis Low socioeconomic status ( $\leq$ RM 2300 or 531 USD), Middle socioeconomic status (RM 2301-5600 or 531-1294 USD) and High socioeconomic status (> RM 5600 or 1294 USD)

OR Odds ratio, $\mathrm{Cl}$ confidence interval, $B M I$ Body mass index, NGO Non-governmental organization

Other comorbidities: Blood clots, depression, asthma, osteoarthritis, pregnancy losses/birth defects and osteoporosis

was less common in married people which were undergoing dialysis therapy while divorced/widowed patients were at higher risk of depression [52]. Similar results were reported from a study in Taiwan where the status of marriage in HD patients was significantly associated with better quality of life [58]. On the other hand, Kimmel and colleagues reported that rate of depression is higher among divorced and widowed women and depression is associated with patient's poor quality of life [6]. Supportive and peaceful family environment, happy married life and family support is associated with depression free and better quality of life in chronic HD patients [24]. These findings are in contradiction to the findings of the current study.

Out of the total 140 non-smokers in our study, 85.7\% patients were having depression. This is in contradiction to the study findings where authors reported that more than half of the current smokers undergoing dialysis therapy were having depression [59]. Beside in dialysis patients, many epidemiological studies have shown that reciprocal relationship exists between smoking and depression [60-62]. In some studies, it has been reported that health related quality of life (HRQoL) was not improved in patients by smoking cessation [63] and depressed smokers have very less chances to quit smoking
[44-66]. Hence, Smoking should be discouraged among HD patients to improve quality of life and to prevent cardiovascular events.

In our study in multivariate logistic regression analysis, treatment given to patients at NGO's running HD centers $(\mathrm{OR}=0.347, p$-value $=0.039)$ had statistically significant negative association with prevalence of depression at final visit. Dalrymple et al. found that overall hospitalization rates of HD patients were remarkably higher $(15 \%$ higher) for those patients which were receiving treatment in for-profit HD facilities as compared with nonprofit dialysis centers [67]. In Malaysia, the government is the main source of funding for new and existing patients on dialysis. There are 3 different sectors i.e. government, NGO's and private dialysis centers that are providing dialysis treatment to patients in Malaysia. These funds provided by government are not only allocated for government dialysis facilities but also for NGOs running centers, for public pensioners, civil servants and their family members who are undergoing dialysis therapy in private dialysis facilities. Self-funding for dialysis treatment had dropped remarkably from $26 \%$ in 2006 to $17 \%$ in 2015. Increase in funding from NGO bodies from $12 \%$ in 2006 to $15 \%$ in 2015 was reported [68]. It is reported that in economically advanced states 
of Malaysia, patients were taking dialysis treatment in NGOs running centers and in private dialysis centers and the survival rates and quality of life of HD patients were better as compared to public dialysis centers. On the other hand, in states like Sabah, Sarawak, Kelantan and Terengganu $50 \%$ of patients were taking dialysis treatment in public sector dialysis facilities [69]. NGOs running programs like Syrian American Medical Society (SAMS) was initiated to help the Syrian patients in refugee camps and northern Syria during the crises in Syria. SAMS was basically a mission of Syrian American nephrologists for the direct observation, to treat psychological disorders and care of dialysis patients which was severely compromised due to destruction of health care facilities, loss of access to dialysis centers, lack of medications and sue to shortage of medical care professionals [70]. But in another study on assessment of ESRD during Syrian crises, the authors found that the aid from inexperienced NGOs and non-renal charities despite of their good will is insufficient and potentially dangerous. Regional and international renal teams should be involved in organizing aid in situations like Syrian crises [71]. A significant improvement in mortality rate over the years and reduce hospitalization rates due to providing adequate dialysis therapy, EPO and iron usage was reported in NGO based dialysis center. Moreover, the free supply of antihypertensive drugs was associated with better control of hypertension and reduced rates of cardiovascular mortality at this NGO funded dialysis facility in south India [72]. Authors of a study reported that Malaysian government reforms to encourage NGOs dialysis facilities and private facilities has brought a transformation and resulted in greatly expanded and an easy access to dialysis patients specially with low socioeconomic status to avail dialysis services [73]. Those dialysis patients who were receiving financial help from NGO's, hospitals and other funding organizations were less depressed as compared to those who were not [74]. Most notably, the association of depression in NGOs and government sector dialysis centers has never been studied. Further studies are warranted to confirm this finding.

\section{Strengths and limitations of the study}

- This study involved a group of patients from tertiary-level teaching hospital of Malaysia.

- To the best of the authors' knowledge, this is the first follow up study to assess the prevalence and predictors of depression among hemodialysis patients in a Malaysian setting.

- For determining the factors associated with depression, multivariate analysis was conducted.

- Being a prospective observational study, the findings of the present study need to be interpreted with caution since it is limited to only 6 months follow up.

- Nevertheless, a multicenter study with a large sample size and longer follow up time is needed to confirm the findings of the current study.

\section{Conclusion}

The current study revealed that the negative association of depression with dialysis therapy at NGOs running dialysis facilities is an indication of better depression management practices at these centers. For better management of depression and to enhance the quality of life of HD patients, studies should be carried out on national level in government, private and NGOs running dialysis centers and strategies should be adopted on how to reduce the prevalence of depression where it is more prevalent.

\section{Study limitations}

The findings of the present study need to be interpreted with caution since it is limited to only 6 months follow up. Nevertheless, a multicenter study with a large sample size and longer follow-up time is needed to confirm the findings of the current study. As we have not correlated the depression scores of same individuals assessed on multiple times, our results should be interpreted with the limitation of separate analysis.

\section{Abbreviations \\ ADNAN: Advanced Dialysis Nephrology Application Network; BDI: Beck's Depression Inventory; CKD: Chronic kidney disease; ESRD: End stage renal disease; HADS: Hospital anxiety and depression scale; HD: Hemodialysis; HRQOL: Health related quality of life; HUSM: Hospital University Sains Malaysia; NGOs: Non-governmental organizations; PD: Peritoneal dialysis; QOL: Quality of life; WHO: World health organization}

\section{Acknowledgments}

We are grateful to the Institute of Postgraduate Studies (IPS) of Universiti Sains Malaysia (USM) for the fellowship support [Ref. no. P-FD0011/15(R)].

\section{Funding}

This research received no specific grant from any funding agency in the public, commercial or not-for-profit sectors.

\section{Availability of data and materials}

All data generated or analyzed during this study are included in this current article. The datasets used and/or analyzed during the current study are available from the corresponding author on reasonable request.

\section{Authors' contributions}

All authors (AK, AHK, ASA, SASS, SM) made substantial contributions to the conception and design of this study. AK and AHK made substantial contributions to the acquisition and analysis of the data. AK drafted the manuscript and ASA, SASS, and SM were involved in critical revision for important intellectual content. All authors read and approved the final manuscript.

\section{Ethics approval and consent to participate}

The current study was approved by the Human Resource Ethics Committee of Hospital Universiti Sains Malaysia (USM/JEPeM/16020058). Written informed consent was obtained from all individual participants included in the study. All procedures performed in studies involving human participants were in accordance with the ethical standards of the institutional and/or national 
research committee and with the 1964 Helsinki declaration and its later amendments or comparable ethical standards.

\section{Consent for publication}

Not applicable.

\section{Competing interests}

The authors declare that they have no competing interests.

\section{Publisher's Note}

Springer Nature remains neutral with regard to jurisdictional claims in published maps and institutional affiliations.

\section{Author details}

${ }^{1}$ Discipline of Clinical Pharmacy, School of Pharmaceutical Sciences, Universiti Sains Malaysia, 11800 Penang, Malaysia. ${ }^{2}$ Chronic Kidney Disease Resource Centre, School of Medical Sciences, Health Campus, Universiti Sains Malaysia, 16150 Kubang Kerian, Kelantan, Malaysia. ${ }^{3}$ Department of Pharmacy, Quaid-i-Azam University, Islamabad 45320, Pakistan. ${ }^{4}$ Health Care Biotechnology Department, Atta ur Rahman School of Applied Biosciences, National University of Science \& Technology, Islamabad 44000, Pakistan. ${ }^{5}$ Management Science University, University Drive, Off Persiaran Olahraga, Section 13, 40100 Shah Alam, Selangor, Malaysia.

Received: 1 September 2018 Accepted: 10 April 2019 Published online: 09 May 2019

\section{References}

1. WHO report 2018. Retrieved from http://www.who.int/news-room/factsheets/detail/depression.

2. Turkistani I, et al. The prevalence of anxiety and depression among endstage renal disease patients on hemodialysis in Saudi Arabia. Ren Fail. 2014; 36(10):1510-5.

3. Wa Wang L-J, Chen C-K. The Psychological impact of hemodialysis on patients with chronic renal failure. In: Renal failure-the facts; 2012. InTech.

4. Van Biesen W, et al. Fluid status in peritoneal dialysis patients: the European body composition monitoring (EuroBCM) study cohort. PLoS One. 2011;6(2): e17148.

5. Chertow GM, et al. Bioimpedance norms for the hemodialysis population. Kidney Int. 1997;52(6):1617-21.

6. Kimmel PL, et al. ESRD patient quality of life: symptoms, spiritual beliefs, psychosocial factors, and ethnicity. Am J Kidney Dis. 2003;42(4):713-21 91 \& $126 \& 285$.

7. Stasiak CES, et al. Prevalence of anxiety and depression and its comorbidities in patients with chronic kidney disease on hemodialysis and peritoneal dialysis. J Bras Nefrol. 2014;36(3):325-31.

8. Wu AW, et al. Changes in quality of life during hemodialysis and peritoneal dialysis treatment: generic and disease specific measures. J Am Soc Nephrol. 2004;15(3):743-53

9. Ginieri-Coccossis M, et al. Quality of life, mental health and health beliefs in haemodialysis and peritoneal dialysis patients: investigating differences in early and later years of current treatment. BMC Nephrol. 2008;9(1):14.

10. Varela $L$, et al. Predictores psicológicos de la calidad de vida relacionada con la salud en pacientes en tratamiento de diálisis peritoneal. Nefrología (Madrid). 2011;31(1):97-106.

11. Zigmond A, Snaith R. The hospital anxiety and depression scale. Acta Psychiatr Scand. 1983;67(6):361-70.

12. Whelan-Goodinson R, Ponsford J, Schönberger M. Validity of the hospital anxiety and depression scale to assess depression and anxiety following traumatic brain injury as compared with the structured clinical interview for DSM-IV. J Affect Disord. 2009;114(1):94-102.

13. Yahya F, Othman Z. Validation of the Malay version of hospital anxiety and depression scale (HADS) in Hospital Universiti Sains Malaysia. Int Med J. 2015;22:80-2

14. Bjelland I, et al. The validity of the hospital anxiety and depression scale: an updated literature review. J Psychosom Res. 2002;52(2):69-77.

15. Maldonado G, Greenland S. Simulation study of confounder-selection strategies. Am J Epidemiol. 1993;138(11):923-36.

16. Bossola M, et al. Correlates of symptoms of depression and anxiety in chronic hemodialysis patients. Gen Hosp Psychiatry. 2010;32(2):125-31 168 $\& 192$.
17. Taskapan $\mathrm{H}$, et al. Psychiatric disorders and large interdialytic weight gain in patients on chronic haemodialysis. Nephrology. 2005;10(1):15-20.

18. Drayer RA, et al. Characteristics of depression in hemodialysis patients: symptoms, quality of life and mortality risk. Gen Hosp Psychiatry. 2006;28(4):306-12.

19. Kalender B, et al. Quality of life in chronic kidney disease: effects of treatment modality, depression, malnutrition and inflammation. Int J Clin Pract. 2007;61(4):569-76.

20. Cukor $\mathrm{D}$, et al. Course of depression and anxiety diagnosis in patients treated with hemodialysis: a 16-month follow-up. Clin J Am Soc Nephrol. 2008;3(6):1752-8.

21. Hedayati SS, et al. Death or hospitalization of patients on chronic hemodialysis is associated with a physician-based diagnosis of depression. Kidney Int. 2008;74(7):930-6.

22. Ibrahim S, El Salamony O. Depression, quality of life and malnutritioninflammation scores in hemodialysis patients. Am J Nephrol. 2008;28(5):784-91.

23. Hsu HJ, Chen C-K, Wu M-S. Lower prevalence of depression in hemodialysis patients who use polysulfone dialyzers. Am J Nephrol. 2009;29(6):592-7.

24. Kao TW, et al. Economic, social, and psychological factors associated with health-related quality of life of chronic hemodialysis patients in northern Taiwan: a multicenter study. Artif Organs. 2009;33(1):61-8 254 \& 286.

25. Montinaro $V$, et al. Emotional symptoms, quality of life and cytokine profile in hemodialysis patients. Clin Nephrol. 2010;73(1):36.

26. Preljevic VT, et al. Screening for anxiety and depression in dialysis patients: comparison of the hospital anxiety and depression scale and the Beck depression inventory. J Psychosom Res. 2012;73(2):139-44 (171 \& 195).

27. Tavallaii SA, et al. Effect of depression on health care utilization in patients with end-stage renal disease treated with hemodialysis. Eur J Intern Med. 2009;20(4):411-4 174 \& 198.

28. Keskin $\mathrm{G}$, Engin $\mathrm{E}$. The evaluation of depression, suicidal ideation and coping strategies in haemodialysis patients with renal failure. J Clin Nurs. 2011;20(19-20):2721-32.

29. Araujo SM, et al. Risk factors for depressive symptoms in a large population on chronic hemodialysis. Int Urol Nephrol. 2012;44(4):1229-35.

30. Farrokhi F, et al. Association between depression and mortality in patients receiving long-term dialysis: a systematic review and meta-analysis. Am J Kidney Dis. 2014;63(4):623-35.

31. Boulware $L E$, et al. Temporal relation among depression symptoms, cardiovascular disease events, and mortality in end-stage renal disease: contribution of reverse causality. Clin J Am Soc Nephrol. 2006;1(3):496-504.

32. Kimmel PL, et al. Multiple measurements of depression predict mortality in a longitudinal study of chronic hemodialysis outpatients. Kidney Int. 2000; 57(5):2093-8.

33. Van den Beukel TO, Verdujin M, Boeschoten EW, Krediet RT, Dekker FW, Van Dijk S. The association of baseline, newonset, and persistent depressive symptoms with mortality in ESRD patients. In: Proceedings of the American Society of Nephrology Renal Week; November 16-21, 2010; Denver, CO. Abstract TH-PO529.

34. Hedayati SS, et al. Association between major depressive episodes in patients with chronic kidney disease and initiation of dialysis, hospitalization, or death. Jama. 2010;303(19):1946-53.

35. Tsai Y-C, et al. Association of symptoms of depression with progression of CKD. Am J Kidney Dis. 2012;60(1):54-61.

36. Chilcot J, et al. Depression on dialysis. Nephron Clin Pract. 2008;108(4):c256-64.

37. Kiecolt-Glaser JK, Glaser R. Depression and immune function: central pathways to morbidity and mortality. J Psychosom Res. 2002;53(4):873-6.

38. Bilgic A, et al. Nutritional status and depression, sleep disorder, and quality of life in hemodialysis patients. J Ren Nutr. 2007;17(6):381-8.

39. Cukor $D$, et al. Depression is an important contributor to low medication adherence in hemodialyzed patients and transplant recipients. Kidney Int. 2009;75(11):1223-9.

40. Cuijpers P, Smit F. Excess mortality in depression: a meta-analysis of community studies. J Affect Disord. 2002;72(3):227-36.

41. Schoevers $\mathrm{R}$, et al. Depression and excess mortality: evidence for a dose response relation in community living elderly. Int. J. Geriatr. Psychiatry. 2009; 24(2):169-76.

42. Lopes AA, et al. Screening for depression in hemodialysis patients: associations with diagnosis, treatment, and outcomes in the DOPPS. Kidney Int. 2004:66(5):2047-53.

43. Hedayati $\mathrm{S}$, et al. The predictive value of self-report scales compared with physician diagnosis of depression in hemodialysis patients. Kidney Int. 2006; 69(9):1662-8. 
44. Blumenfield $M$, et al. Fluoxetine in depressed patients on dialysis. Int. J. Psychiatry Med. 1997;27(1):71-80.

45. Spigset $\mathrm{O}$, et al. Citalopram pharmacokinetics in patients with chronic renal failure and the effect of haemodialysis. Eur J Clin Pharmacol. 2000;56(9-10): 699-703.

46. Wuerth, D., S.H. Finkelstein, and F.O. Finkelstein. Psychosocial factors in patients with chronic kidney disease: the identification and treatment of depression in patients maintained on dialysis. In Seminars in dialysis. 2005. Wiley Online Library.

47. Duarte PS, et al. Cognitive-behavioral group therapy is an effective treatment for major depression in hemodialysis patients. Kidney Int. 2009; 76(4):414-21.

48. Ouzouni $\mathrm{S}$, et al. Effects of intradialytic exercise training on healthrelated quality of life indices in haemodialysis patients. Clin Rehabil. 2009;23(1):53-63.

49. Rabindranath KS, et al. Physical measures for treating depression in dialysis patients. Cochrane Database Syst Rev. 2005;1(2):4-9.

50. Nagler EV, et al. Antidepressants for depression in stage 3-5 chronic kidney disease: a systematic review of pharmacokinetics, efficacy and safety with recommendations by European renal best practice (ERBP). Nephrol Dial Transplant. 2012;27(10):3736-45.

51. Nolen-Hoeksema S. Gender differences in depression. Curr Dir Psychol Sci. 2001;10(5):173-6.

52. Theofilou P. Depression and anxiety in patients with chronic renal failure: the effect of sociodemographic characteristics. Int J Nephrol. 2011;2011: 169-93.

53. Lopes GB, et al. Depression as a potential explanation for gender differences in health-related quality of life among patients on maintenance hemodialysis. Nephron Clinical Practice. 2010;115(1):c35-40.

54. House AA, et al. Definition and classification of cardio-renal syndromes: workgroup statements from the 7th ADQI consensus conference. Nephrol Dial Transplant. 2010;25(5):1416-20.

55. Dohrenwend BP, et al. Socioeconomic status and psychiatric disorders: the causation-selection issue. Science. 1992;255(5047):946-52.

56. Miech RA, Shanahan MJ. Socioeconomic status and depression over the life course. J Health Soc Behav. 2000;41(2):162-76.

57. Link BG, Lennon MC, Dohrenwend BP. Socioeconomic status and depression: the role of occupations involving direction, control, and planning. Am J Sociol. 1993;98(6):1351-87.

58. Chiang C-K, et al. Health-related quality of life of hemodialysis patients in Taiwan: a multicenter study. Blood Purif. 2004;22(6):490-8.

59. Østhus TBH, et al. Health-related quality of life and depression in dialysis patients: associations with current smoking. Scand J Urol Nephrol. 2010; 44(1):46-55.

60. Egede LE, Zheng D. Independent factors associated with major depressive disorder in a national sample of individuals with diabetes. Diabetes Care. 2003;26(1):104-11.

61. Breslau N, et al. Major depression and stages of smoking: a longitudinal investigation. Arch Gen Psychiatry. 1998;55(2):161-6.

62. Pasco JA, et al. Tobacco smoking as a risk factor for major depressive disorder: population-based study. Br J Psychiatry. 2008;193(4):322-6.

63. Sarna $L$, et al. Impact of smoking and smoking cessation on health-related quality of life in women in the nurses' health study. Qual Life Res. 2008; 17(10):1217-27.

64. Anda RF, et al. Depression and the dynamics of smoking: a national perspective. Jama. 1990;264(12):1541-5.

65. Glassman $\mathrm{AH}$, et al. Smoking, smoking cessation, and major depression. Jama. 1990;264(12):1546-9.

66. Thorndike AN, et al. Depressive symptoms and smoking cessation after hospitalization for cardiovascular disease. Arch Intern Med. 2008;168(2):186-91.

67. Straube BM. Do health outcomes vary by profit status of hemodialysis units? Clin J Am Soc Nephrol. 2014;9(1):1-2.

68. $23^{\text {rd }}$ Report of the Malaysian Dialysis and Transplant Registry 2015. Retrieved from https://www.msn.org.my/nrr/mdtr2015.jsp.

69. Chan YM, Zalilah MS, Hii SZ. Determinants of compliance behaviours among patients undergoing hemodialysis in Malaysia. PLoS One. 2012;7(8):e41362.

70. Al-Makki A, et al. The Syrian national kidney foundation: response for the need of kidney patients during the crisis. Avicenna J Med. 2014;4(3):54.

71. Sekkarie MA, et al. The Syrian conflict: assessment of the ESRD system and response to hemodialysis needs during a humanitarian and medical crisis. Kidney Int. 2015;87(2):262-5.
72. Georgi A, et al. Resource settings have a major influence on the outcome of maintenance hemodialysis patients in South India. Hemodial Int. 2010;14(2): $211-7$.

73. Lim T-O, et al. How public and private reforms dramatically improved access to dialysis therapy in Malaysia. Health Aff. 2010;29(12):2214-22.

74. Anees M, et al. Depression in hemodialysis patients. Pak J Med Sci. 2008; 24(4):560-5.

\section{Ready to submit your research? Choose BMC and benefit from:}

- fast, convenient online submission

- thorough peer review by experienced researchers in your field

- rapid publication on acceptance

- support for research data, including large and complex data types

- gold Open Access which fosters wider collaboration and increased citations

- maximum visibility for your research: over $100 \mathrm{M}$ website views per year

At BMC, research is always in progress.

Learn more biomedcentral.com/submissions 